\title{
Multiplicity Sorter for Shift-Register \\ Coincidence Electronics
}

J. E. Swansen

P. R. Collinsworth

M. S. Krick

D. L. Peterson 
MULTIPLICITY SORTER FOR SHIFT-REGISTER COINCIDENCE ELECTRONICS

by

J. E. Swansen, P. R. Collinsworth,

M. S. Krick, and D. L. Peterson

\begin{abstract}
A multiplicity sorter for the latest Los Alamos Nationa? Laboratory version of shift-register coincidence electronics is described. The multiplicity information taken from the coincidence-gate up-down counter is decoded and gated onto the output lines by prompt real-plus-accidental $(R+A)$ and delayed accidental (A) strobes. Multiplicities of $0-7$ and $\geq 8$ are sorted for hoth the $\mathrm{P}+\mathrm{A}$ and $\mathrm{A}$ gates.
\end{abstract}

\title{
I. INTRODUCTION
}

The latest Los Alamos National Laboratory shift-register coincidence electronics system for thermal neutron counters has been described in detail.1,2 For studies of neutron multiplication, it is desirable to measure the coincidence gate multiplicity distributions. ${ }^{3}$

A block diagram of the coincidence circuit and the multiplicity sorter is shown in Fig. 1. Every incoming pulse effectively opens two coincidence gates, one prompt and one delayed. The contents of the gates are monitored by the up-down counter. The prompt input pulse strobes the conterits of the up-down counter into the real-plus-accidental $(R+A)$ accumulator; after $1024 \mu \mathrm{s}$, the delayed input pulse strobes the contents of the up-down counter into the accidental (A) accumulator.

The multiplicity distributions are stained by decoding the contents of the up-down counter and gating the output lines with the $R+A$ and $A$ strobes. The lower six rits of the up-down counter, the $R+A$ strobe, and the $A$ strobe are buffe:ed in the coinciderice electronics package and are brought out on a ribbon 
cable to a multiplicity decoding package, which is a custom-wired, double-width NIM module (Fig. 2).

After additional buffering in the nuclear instrument module (NIM), the multiplicity data are decoded and strobed onto nine output 1 ines $(0-7$ and $\geq 8)$ for both the $P+A$ and $A$ gates. These 18 output lines are wired to LEMO connectors on the front panel of the NIM module.

A block diagram of the experimental multiplicity counting system is shown in Fig. 3. The 18 multiplicity output lines are connected to 3 CAMAC hex scaiers (Kinetic Systems 3615) controlled by a Kinetic jystems 3988 crate controller. The entire system is under the control of an HP-85 desk-top computer, which is interfaced to the CAMAC crate on an HP-IB bus and is interfaced to the coincidence electronics package on an $R_{i}-23$ ? line. The coincidence package has the new version of the PROM software that allows bidirectional communication on the PS-232. 1ine. 4

\section{A. Circuitry}

A schematic of the buffer circuitry added to the coincidence electronics package is shown in Fig. 4. Three sockets were installed in spare locations on the shift-register circuit board. Two of these hold 74LS366 hex drivers; the other is the socket for a 3-m-long, 16-wire ribbon cable that connects the coincidence package to the multiplicity decoding package.

The designations $3 \mathrm{C}, 2 \mathrm{~B}$, etc, , refer to the integrated circuit location on the shift-register board. The pin numbers are indicated beside each wire. A schematic of the original shift-register board is shown in Ref. 5 (Dwg. No. 0200734 ).

The up-down counter consists of two decade counters; the lower six bits therefore carry a maximum count of 39 . (It was assumed that multiplicities $>39$ would be negligible.) The six bits corresponding to the $1,2,4,8,10$, and 20 lines plus the $R+A$ and $A$ strobes are buffered and inverted by the 74 LS366 drivers before heing sent to the decoding module. The ribbon cable used for the transfer is wired for alternate signal and ground wires and is brought out of the coincidence package just under the top cover plate.

A schematic of the multiplicity decoding module is shown in Fig. 5 . The eight incoming signal lines are terminated with $330-\Omega$ resistors. All but the $\overline{10}$ and $\overline{20}$ lines are huffered by 74 LS14 hex Schmitt-trigger inverters, whereas the 10 and $\overline{20}$ lines are huffered by a $74 L S 132$ quad Schmitt-trigger NAND gate. 
Two 74LS174 hex D-type flip-flops latch the multiplicity data when clocked by the $R+A$ and $A$ strohes; the latches store the multiplicity data for the duration of the $R+A$ and $A$ strobe pulses. After a 100-ns delay to allow the outputs of the latches to settle, the R+A and $A$ strobes gate on the 74LS138 3-to-8 line decoders. Because the LST38s decode the 1, 2, and 4 lines to $0-7$, multiplicities $\geq 8$ must inhibit the decoder; this is accomplished by using the 8,10 , or 20 signals. Two 74 LS240 octal buffers are used as line drivers for the $0-7$ multiplicity pulses, which are sent through $50-\Omega$ resistors to front-pane 1 LEMO connectors for transfer through coaxial cables to the multiplicity scaters.

Two 74LS28 quad NOR buffers generate outputs for multiplicities $\geq 8$ and $\leq 39$. The output buffer is enabied whenever 8,10 , or 20 is logica 1 ; the $R+A$ and $A$ strobes then produce the output pulses to the LEMO connecturs.

The NIM module is powered from the $+6 V$ supply; the $+5 V$ supply voltage is obtained from an MJE3055 operated as a power diode.

\section{B. Measurements}

Table I shows a multiplicity measurement made with a $\mathrm{PuO}_{2}$ sample in a thermal neutron counter. The total neutron counting rate was $29250 \mathrm{pps}$ and the coincidence gate length was $32 \mu \mathrm{s}$. The time, total counts, $R+A$ coincidence counts, and $A$ coincidence counts are from the standard coincidence electronics package; the multiplicities are from the new multiplicity sorter and CAMAC scalers. The maximum count per channel in the Kinetic Systems 3615 hex scalers is $2^{24}$, so the overflows were scaled with HP-85 software.

Because every input pulse produces an $R+A$ and $A$ coincidence strobe, the sum of the multiplicity counts for either the $R+A$ or $A$ distributions must equal the total counts if the system is working correctly. This is easily verified for the example in Table I. No failure of this test has occurred in the first 6 montlis of operation.

\section{Conclusions}

The multiplicity sorter can be used to obtain reliable multiplicity data from shift-register coincidence electronics, with minor modification of the original shift-register board. The decoding, scaling, and readout circuitry can probabiy be packaged in a NIM module to avoid the use of a CAMAC system. 
Measurement time $(s)$
Total counts
$R+A$ counts
A counts

Multiplicity

1

3

4

5

6

7

$\geq 8$
0

2

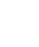

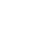

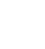

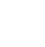

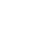

7

7

8

1

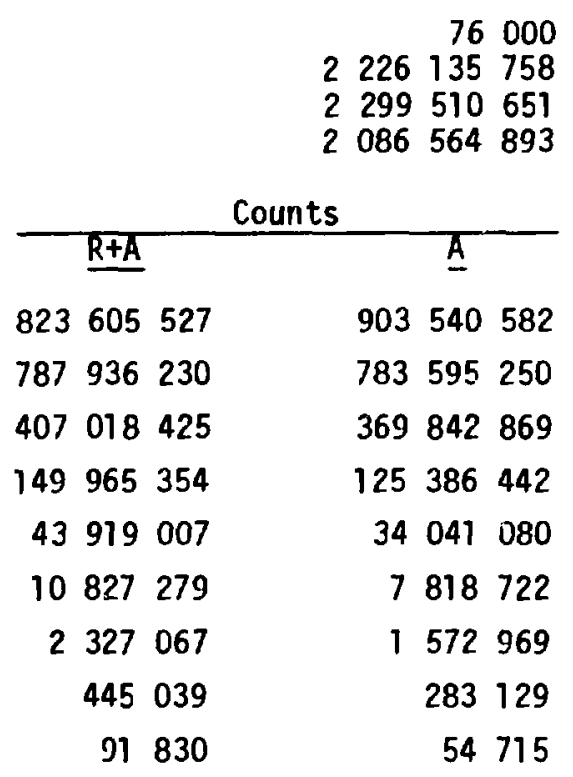

\section{REFERENCES}

1. J. E. Swansen, P. R. Collinsworth, and M. S. Krick, "Shift-Register Coincidence Electronics System for Thermal Meutron Counters," Los Alamos Scientific Laboratory report LA-8319-inS (Apri1 1980).

2. J. E. Swansen, P. R. Collinsworth, and M. S. Krick, "Shift-Register Coincidence Electronics System for Thermal Neutron Counters," Nuc1. Instrum. Methods 176, 555-565 (1980).

3. M. S. Krick. "Neutron Multiplication Corrections for Passive Thermal Neutron Wall Counters," Los Alamos Scientific Laboratory report LA-8460-MS (July 1980).

4. J. E. Swansen, "A New 1-k PROM for the Coincidence Counter Electronics Package," to be published as a Los Alamos National Laboratory report.

5. D. Altman, "High Level Neutron Coincidence Counter (HLNCC): Maintenance Manual," EC\&C report EGG-1183 5091 (Ju1y 1978). 


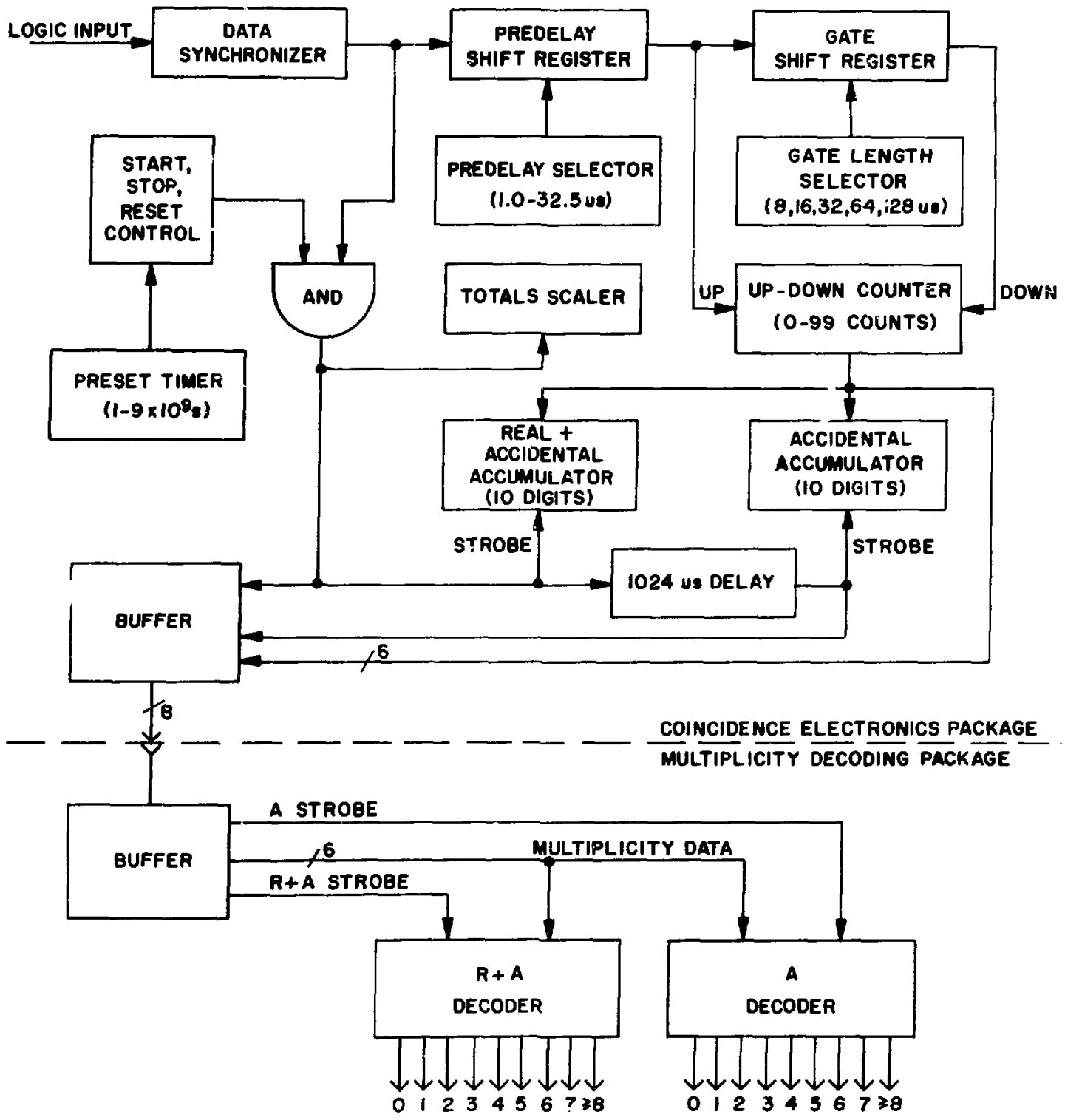

Fig. 1 .

Block diagram of shift-register coincidence circuit and multiplicity sorter. 


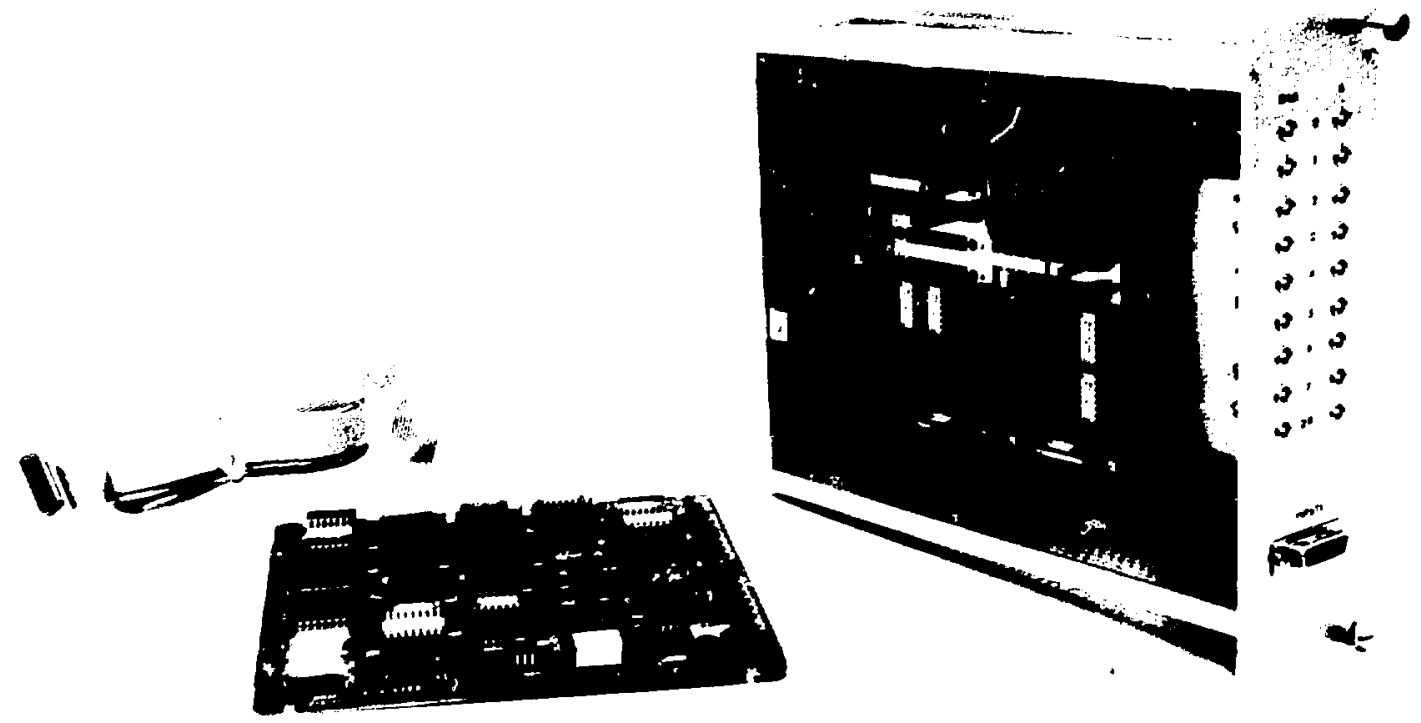

Fig. 2 .

Modified shift-register board with cable and custom-wired double-width NIM module.

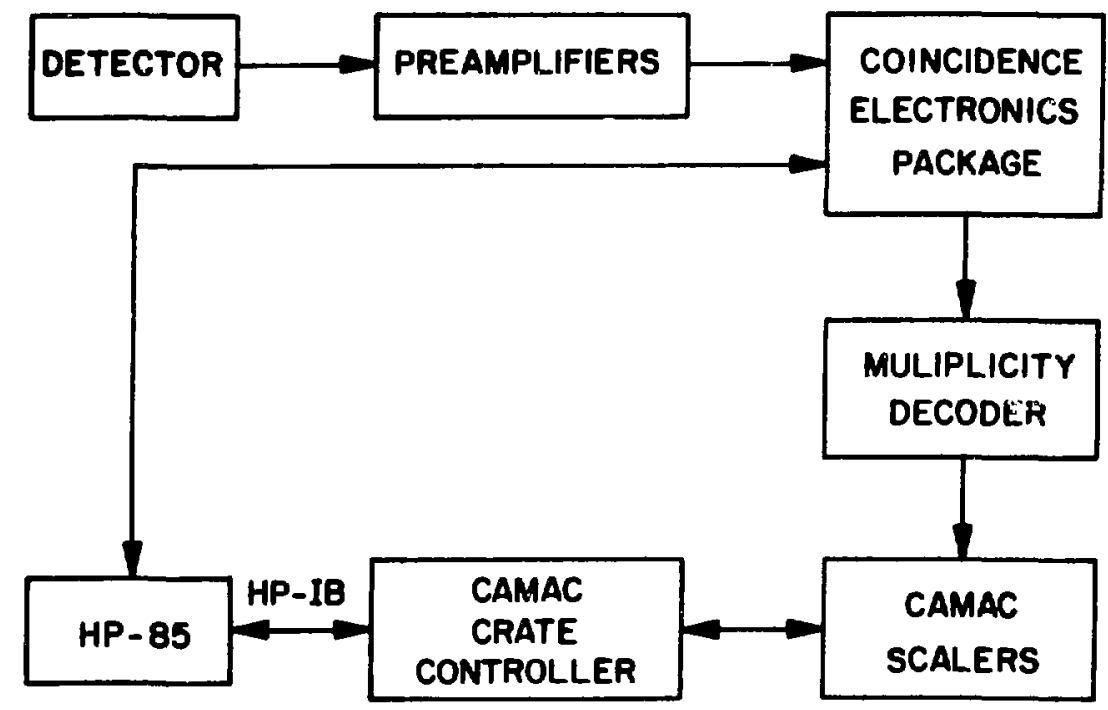

Fig. 3 .

Block diagram of experimental multiplicity counting system. 


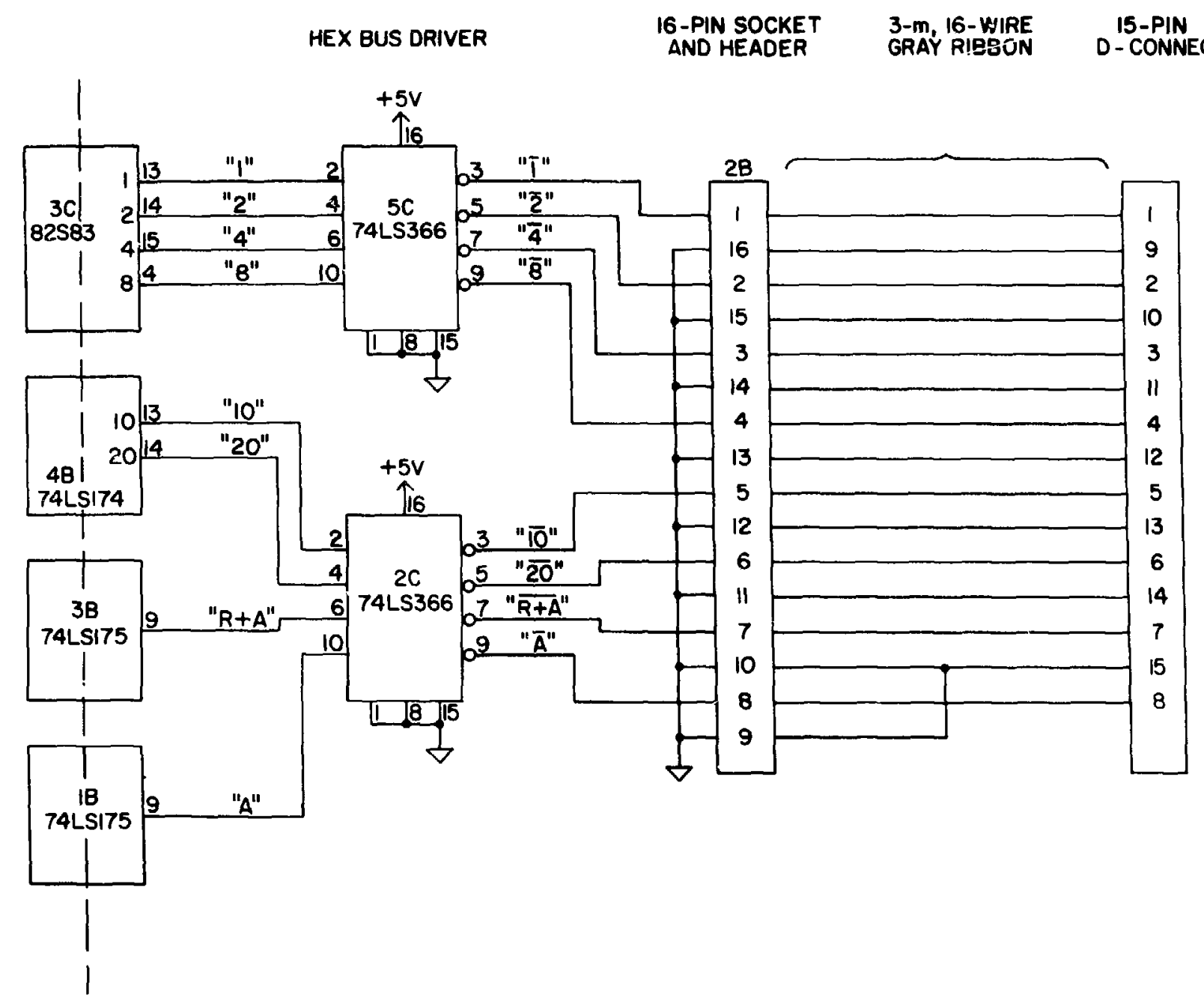

Fig. 4. 


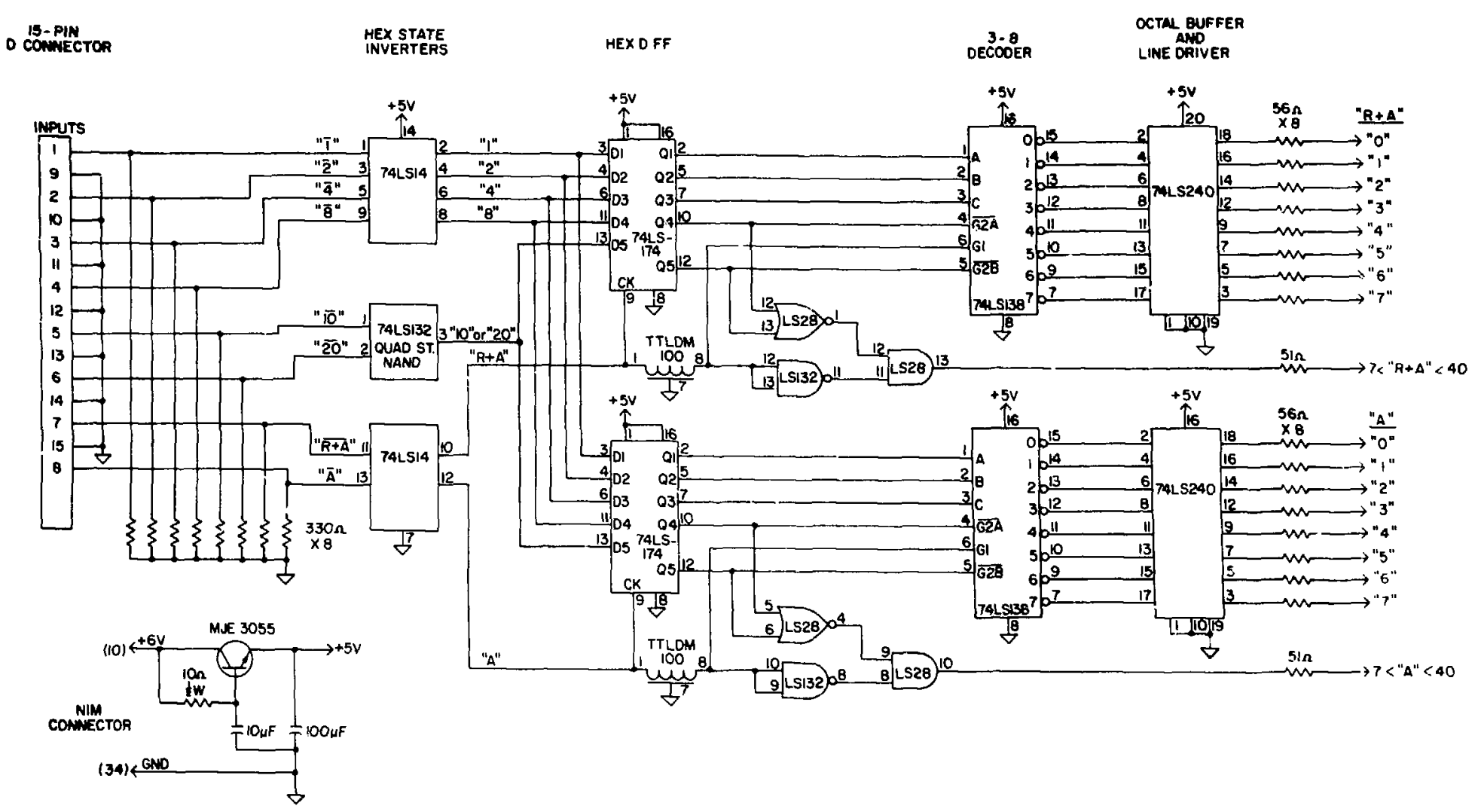

Fig. 5.

Schematic of multiplicity decoding module. 